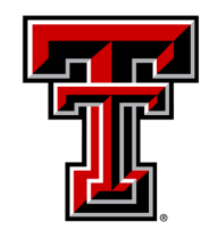

TEXAS TECH UNIVERSITY

Libraries"

\title{
SIMULATIONS OF FIELD EMISSION FROM COPPER ELECTRODES WITH INCLUSION OF OXYGEN SURFACE LAYER AND WORK FUNCTION CHANGES BASED ON FIRST-PRINCIPLES CALCULATIONS
}

\section{The Texas Tech community has made this publication openly available. Please share how this access benefits you. Your story matters to us.}

\begin{tabular}{|l|l|}
\hline Citation & $\begin{array}{l}\text { S. N. Sami, L. Diaz, M. Sanati, and R. P. Joshi, "Simulations of Field } \\
\text { Emission from Copper Electrodes with Inclusion of Oxygen Surface } \\
\text { Layer and Work Function Changes Based on First-Principles } \\
\text { Calculations," Journ. Applied Physics 128, 223302 (2020). } \\
\text { https://doi.org/10.1063/5.0031568 }\end{array}$ \\
\hline Citable Link & $\underline{\text { https://hdl.handle.net/2346/86903 }}$ \\
\hline Terms of Use & $\begin{array}{l}\text { This article may be downloaded for personal use only. Any other } \\
\text { use requires prior permission of the author and AlP Publishing. }\end{array}$ \\
\hline
\end{tabular}




\section{Simulations of field emission from copper electrodes with inclusion of oxygen surface layer and work function changes based on first-principles calculations}

Cite as: J. Appl. Phys. 128, 223302 (2020); https://doi.org/10.1063/5.0031568

Submitted: 01 October 2020 . Accepted: 25 November 2020 . Published Online: 10 December 2020

S. N. Sami, (iD) L. Diaz, M. Sanati, and (D. R. P. Joshi
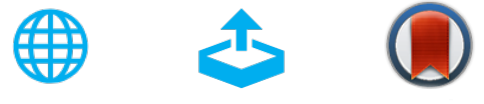

View Online

Export Citation

\section{ARTICLES YOU MAY BE INTERESTED IN}

Analysis of energy exchanges during the interaction between pulsed lightning arcs and metallic plates

Journal of Applied Physics 128, 223301 (2020); https://doi.org/10.1063/5.0025616

Coupled analysis to probe the effect of angular assignments on the secondary electron yield (SEY) from copper electrodes

Physics of Plasmas 27, 093511 (2020); https://doi.org/10.1063/5.0010389

Transitions between electron emission and gas breakdown mechanisms across length and pressure scales

Journal of Applied Physics 128, 210903 (2020); https://doi.org/10.1063/5.0030220

Challenge us. What are your needs for periodic signal detection?

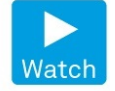

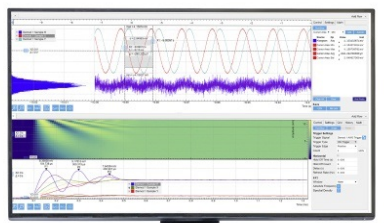

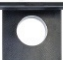

Zurich - Instruments 


\title{
Simulations of field emission from copper electrodes with inclusion of oxygen surface layer and work function changes based on first-principles calculations
}

Cite as: J. Appl. Phys. 128, 223302 (2020); doi: 10.1063/5.0031568

Submitted: 1 October 2020 - Accepted: 25 November 2020 .

Published Online: 10 December 2020

S. N. Sami, ${ }^{1}$ (D) L. Diaz, ${ }^{2}$ (D) M. Sanati, ${ }^{2}$ and R. P. Joshi ${ }^{1, a)}$ (D)

\begin{abstract}
AFFILIATIONS
${ }^{1}$ Department of Electrical and Computer Engineering, Texas Tech University, Lubbock, Texas 79409, USA

${ }^{2}$ Department of Physics and Astronomy, Texas Tech University, Lubbock, Texas 79409, USA
\end{abstract}

${ }^{a)}$ Author to whom correspondence should be addressed: ravi.joshi@ttu.edu

\begin{abstract}
Analysis of field emission requires the inclusion of the internal potentials that shape the electronic wavefunctions and tunneling probabilities; details of the work function that are dependent on material quality and defects; and the role of the density of states (DOS) that influences the electronic supply. Here, these factors are collectively included on the basis of density functional theory to obtain predictions of field-dependent electron tunneling current densities. Results are obtained in copper for three different orientations. The DOS is predicted to be broadened by an externally applied electric field. The (100) copper is shown to yield the largest current density, and the (111) orientation is the lowest. The presence of an oxide surface monolayer is shown to increase the work function, leading to the emission of current reductions. The technique is general and can be applied to other materials (e.g., carbon fibers) that have shown promise as cathode emitters.
\end{abstract}

Published under license by AIP Publishing. https://doi.org/10.1063/5.0031568

\section{INTRODUCTION}

Field emission is the well-known process wherein a strong electric field applied to a metal surface extracts electrons via quantummechanical tunneling. ${ }^{1-9}$ Useful applications include sources for flat and thin displays, $\mathrm{x}$-ray tubes, in vacuum electronics, charged particle generation for microwave devices, in microscopy and lithography, and some medical therapies. ${ }^{10-16} \mathrm{~A}$ benefit of field emission displays would be the lower power consumption over conventional cathode tubes that are based on thermal emission. The ability of field emitters to provide large current densities with high pulse repetition rates is another attractive attribute. A research topic of current interest in this regard centers around large area emitter arrays ${ }^{17-20}$ that offer high brightness, high current density beams with a small spread in energy at low operational temperatures, and high power.

Oppenheimer was the first to invoke electron tunneling (although not directly by name) through a potential barrier to explain electric field induced emission from atoms. ${ }^{21}$ Soon after, several studies on the topic emerged led by the landmark work by
Fowler and Nordheim, ${ }^{1,2}$ the tunneling related $\alpha$-particle decay, ${ }^{22,23}$ and later the notion of field induced electron transport between energy bands in solid dielectrics. ${ }^{24}$ With an evolving understanding of this phenomenon and technological progress, the electronics industry pushed its efforts toward the nanoscale with groundbreaking work in conducting polymers, ${ }^{25,26}$ molecular wires, ${ }^{27}$ and molecular electronic devices. ${ }^{28,29}$ The concept, however, is general and has been to be applied in various fields such as biochemistry. ${ }^{30}$

Techniques for evaluating electron emission are well known, with the Fowler-Nordheim (FN) theory ${ }^{1,2}$ for field emission and the Richardson-Laue-Dushman (RLD) approach ${ }^{31}$ for the treatment of thermionic emission being two common routes. The asymptotic forms of the FN and RLD equations were developed by Murphy and Good ${ }^{32}$ and Herring and Nichols, ${ }^{33}$ respectively. The commonly used form of the FN equation ${ }^{34}$ relies on elliptical integral functions $v(y)$ and $t(y)$. Accurate approximations to these functions have emerged, ${ }^{35,36}$ leading to better representations. More recently, a generalized thermal-field emission equation was 
derived $^{37-39}$ with a transition from a thermal-like emission regime to a field-dominated realm.

In most treatments though, emission currents are evaluated on the basis of the free-electron approximation and use is often made of the Wentzel-Kramers-Brillouin (WKB) method. ${ }^{40}$ Approaches to improving the FN theory have included modifications of the theory that incorporate the image potential, ${ }^{32}$ though still relying on the WKB approximation. To move beyond the free-electron approximation, a triangular representation of the barrier with an adjustable parameter that enabled the solution of Schrodinger's equation in terms of Airy functions, was proposed. ${ }^{41}$ This yielded improvements to the image-potential corrected FN theory and led to a shift of the image plane. In another approach reported by Lang et al., ${ }^{42}$ field emission currents were calculated self-consistently within the density functional theory. ${ }^{43}$ Their model, however, was based on the consideration of two electrodes placed at very short $(\sim 1.6 \mathrm{~nm})$ distances that could lead to errors. Gohda et al. ${ }^{44}$ developed a fully self-consistent method on the basis of density functional theory (DFT). Using this method, they were able to investigate field emission currents from a biased metallic surface with the interior represented by a jellium model.

In this contribution, we probe the electron field emission current densities as a function of external fields from flat copper surfaces. Details of the local density of states at the surface are naturally folded in through the proposed DFT-based approach. Possible field enhancements ${ }^{45-49}$ have been ignored but can easily be included into the present simulations for a single emitter ${ }^{50}$ or multiple emitters. ${ }^{51}$ Electron emission depends on three factors: (i) the tunneling probability that is influenced by details of the electronic wavefunction in the presence of the local potentials, (ii) the work function barrier $\left(\Phi_{B}\right)$ at the boundary that is dependent on material details such as the crystal orientation, the presence of possible defects, etc., and (iii) the density of states (DOS) at the metal surface that influences the supply of electrons. These various factors can collectively be evaluated based on Density Functional Theory (DFT). Such analysis also yields internal potential profiles, from which the electron wavefunctions (and hence the electron density) can be obtained through a solution of the onedimensional Schrödinger equation if atomic details parallel to the surface are ignored. In our calculations, results for the work function and the internal potentials for three crystal orientations of (100), (110), and (111) copper are first obtained. The electron distributions as well as the tunneling probabilities, density of states, and emission currents for the three orientations in pure copper, are subsequently computed. For completeness, the field-dependent current densities for copper having an oxide surface monolayer (ML) are also included, and they provide a useful comparison against defect-free, crystalline copper.

However, it must be pointed out that this contribution presents an initial step toward better analyses, and some issues still remain. For instance, real emitters have a variety of shapes, and so a full three-dimensional (3D) analysis is really needed to fully and accurately model current emission. For example, different emitter geometries can arise depending on the fabrication and material used. These include Spindt-type microtips, ${ }^{52,53}$ pyramidical diamond emitters, ${ }^{54}$ $\mathrm{ZnO}$ nanoemitters, ${ }^{55}$ or carbon nanotubes and fibers. ${ }^{56,57}$ The rounded whisker shape is the closest to the "ideal" field emitter shape; however, this shape is the poorest with regard to thermal stability. In contrast, a triangular shape is poor as a field emitter but provides higher thermal and mechanical stabilities. ${ }^{58}$

Attempts to expand beyond a one-dimensional analysis have included emitter models based on ellipsoidal or hyperbolic geometries, ${ }^{9,59,60}$ or the use of numerical techniques, ${ }^{61,62}$ or shape factors ${ }^{63}$ to determine the electric fields. There are also other issues that can arise. For example, continuous operation of such emitting devices could lead to dynamic changes in the surface smoothness, or evolving dependencies of the emission characteristics due to adsorbed molecules may arise. We have attempted to obliquely address the aspect of adsorbed molecules to a limited degree in this contribution. Another important step would be to incorporate the $3 \mathrm{D}$ analysis into DFT calculations. However, this does present some challenges, since the method usually relies on the use of periodic boundary conditions. ${ }^{64}$ It is also computationally demanding since a repeated calculation of single electron wavefunctions is required to estimate the kinetic energy of non-interacting electrons. The inclusion of defects can give rise to unrealistic periodic geometries, unless adequate care is taken. So, while we are aware of the shortcomings and are attempting to create more realistic emitter models for future study, the present contribution has a one-dimensional limitation.

\section{MODEL DETAILS}

In the present calculation, an approach similar to that proposed by Gohda et al. ${ }^{44}$ was used. A single metallic field emitter was modeled as a semi-infinite jellium, in which positive ions were replaced by a uniform background charge. Thus, the jellium extended for $z \leq z_{\mathrm{min}}$, with the external field assumed to be normal to the emitting surface at $z=z_{\max }>0$ and along the negative $z$ direction. Ignoring the atomic structure in the direction parallel to the surface, the single-particle wavefunction $\psi(\boldsymbol{r})$ can be expressed as $J \psi(r)=\psi(z) \exp \left(i k_{\|} r_{\|}\right)$. This function $\psi(z)$ can be obtained from a solution of the Schrödinger equation: $-\left[\hbar^{2} /\left(2 m^{*}\right)\right] d^{2} \psi(z) / d z^{2}+$ $V(z) \psi(z)=\left[E-\hbar^{2}\left|k_{\|}\right|^{2} /\left(2 m^{*}\right)\right] \psi(z)$. Here, $V(z)$ is the internal potential, $E$ the electron energy, and $\left|k_{\|}\right|$the wavevector parallel to the surface, with $\hbar$ and $m^{*}$ the usually reduced Planck's constant and effective electron mass, respectively.

The one-dimensional Schrödinger equation was solved numerically using the Numerov scheme. ${ }^{65-67}$ In this method, a specialized discretization formula for numerically integrating differential equations of the form: $d^{2} \psi(z) / d z^{2}=f(z) \psi(z)$, is used. For the time-independent Schrödinger equation, $f(z)=-\left[\left(2 m^{*}\right) / \hbar^{2}\right]\{E-$ $\left.\hbar^{2}\left|k_{\|}\right|^{2} /\left(2 m^{*}\right)-V(z)\right\}$, and for uniform grid points $z_{i}$ with spacing $d z$, the recursive sixth order $\left[O(d z)^{6}\right]$ relation then becomes

$$
\psi_{i+1}=\left[\psi_{i-1}\left\{12-(d z)^{2} f_{i-1}\right\}-2 \psi_{i}\left\{5(d z)^{2} f_{i}+12\right\}\right] /\left[(d z)^{2} f_{i+1}-12\right] .
$$

The potential energies, work functions, and charge densities were calculated from first principles. The Vienna $A b$ initio Simulation Package (VASP), a density-functional pseudopotential software tool ${ }^{68-70}$ that relies on the projector augmented-wave method, ${ }^{71,72}$ was used. The calculations were performed within the 
generalized gradient approximation (GGA) to the exchangecorrelation potential as parameterized by Perdew-Burke-Ernzerhof. ${ }^{73}$ VASP was used to simulate a perpendicular electric field outside of the slab where, in the vacuum region, a dipole sheet is created. ${ }^{74,75}$ The plane wave kinetic energy cutoff was set to $600 \mathrm{eV}$ with the $k$-point integration being done by a modified tetrahedron method. ${ }^{76}$ The Brillouin-zone sampling included a $16 \times 16 \times 2$ MonkhorstPack grid and an additional 0.5 mesh shift.

The structures studied in this work consisted of 10-layer slabs of $\mathrm{Cu}$ atoms at three different high symmetry directions of [100], [110], and [111]. For later simulations, an oxygen monolayer (ML) was added to each $\mathrm{Cu}$ surface with the different orientations considered, for probing the impact of surface modification. A lattice constant of $3.63 \AA$ was used for all structures and was calculated from the fcc $\mathrm{Cu}$ bulk structure. The lattice constant was determined by fitting the Murnaghan equation of state with $\mathrm{Cu}$ bulk total energy and volume data. ${ }^{77}$ The first two layers of $\mathrm{Cu}$ surface and $\mathrm{O}$ atoms are fully relaxed, and the total Hellmann-Feynman force for all of the structures was less than $0.01 \mathrm{eV} / \AA$. Dipole corrections were used to eliminate any errors caused by the periodic boundary conditions. ${ }^{78,79}$

Using VASP, the three-dimensional potentials (including the electrostatic, Hartree, and exchange-correlation potentials) were obtained. Using the three-dimensional potential, one can obtain the average planar potential $\bar{V}(z)$ using the following relation:

$$
\bar{V}(z)=\frac{1}{S} \int V(\boldsymbol{r}) d x d y
$$

where $S$ is the area of the unit cell. However, this planar average would still show oscillations in the bulk that can be removed by applying a macroscopic averaging technique, thus replacing the oscillations with a constant value. Here, macroscopic averaging was performed using the following operation:

$$
\overline{\bar{V}}(z)=\frac{1}{L} \int_{-L / 2}^{L / 2} \bar{V}(z) d z,
$$

where $L$ is the length of oscillations in the $z$ direction (normal to the surface). ${ }^{80}$ The work function, $\boldsymbol{\Phi}_{B}$, was calculated using

$$
\Phi_{B}=E_{v}-E_{F},
$$

where $E_{v}$ and $E_{F}$ are the electrostatic potential in the vacuum and the Fermi level, respectively.

\section{RESULTS AND DISCUSSION}

The average planar potential $\bar{V}(z)$ obtained from DFT calculations for a pure copper slab is shown in Fig. 1. Apart from this copper-vacuum system, the potential profile for a copperoxygen-vacuum arrangement is also included in the figure. It should be mentioned that the use of such DFT calculations naturally folds in details of the local density of states (LDOS) at the surface. As can be seen from Fig. 1, the potential far removed from the surface is a periodic function, with Bloch-like oscillations as expected in a perfect lattice. However, as one gets nearer to the

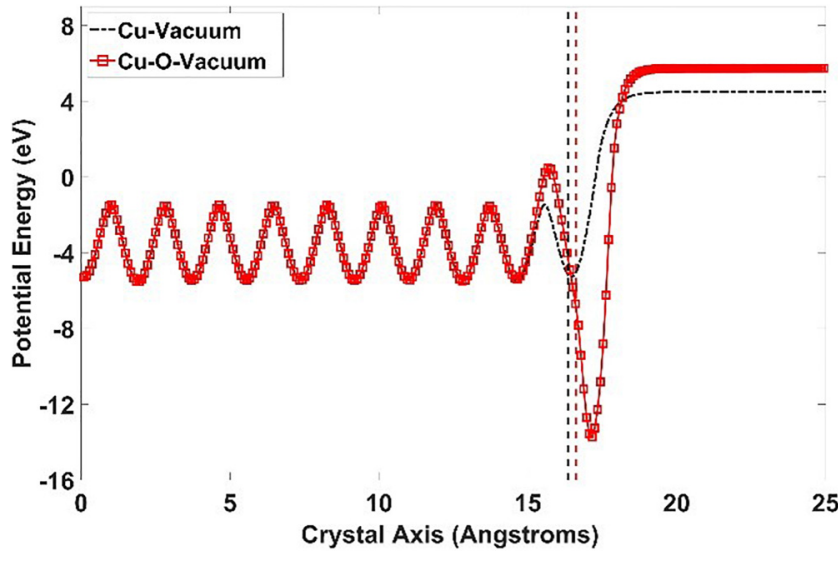

FIG. 1. The average planar potentials $\bar{V}(z)$ obtained from DFT calculations for a pure copper slab and a copper-oxygen-vacuum arrangement. The work function for the $\mathrm{Cu}-\mathrm{O}$ system can be seen to be larger.

surface, the periodic structure is seen to be perturbed. The result for $\bar{V}(z)$ can thus roughly be broken up into three regions: (i) a zone A that exhibits the periodicity over a region deep into the copper. In Fig. 1, this roughly extends from $z<\sim 13.5 \AA=z_{\text {min }}$. (ii) A constant potential over zone $\mathrm{C}$ near the interface and away from the copper surface, beyond about $18 \AA\left(=z_{\max }\right)$, and (iii) an intermediate transition region B roughly between $13.5 \AA$ and $18 \AA$. In the figure, the work function for the $\mathrm{Cu}-\mathrm{O}$ system can be seen to be larger than pure copper.

The averaged potentials $\overline{\bar{V}}(z)$ for pure copper with and without an external DC electric field for the three [100], [110], and [111] directions are shown in Figs. 2(a), 2(b), and 2(c), respectively. The corresponding boundaries $\left(z_{\max }\right)$ in the three figures were located at slightly different positions of $15.5 \AA, \sim 11 \AA$, and $15.5 \AA$. The dashed lines $\left(V_{o}\right)$ in all three cases show the averaged potential in the absence of an external electric field. With the application of an external field (solid lines, $V_{E}$ ) of $5 \mathrm{GV} / \mathrm{m}$, the potential is seen to drop in the vacuum region on the right.

Based on the internal potentials $\overline{\bar{V}}(z)$, the Schrödinger Wave Equation (SWE) was solved to obtain the electronic wavefunctions for the various cases. The solution involved a numerical discretization of the SWE, with the entire region being divided into three zones $\mathrm{A}, \mathrm{B}$, and $\mathrm{C}$. Of these, zone $\mathrm{A}$ was at the leftmost end with a zero averaged potential. Zone $\mathrm{B}$ was the middle region extending beyond zone A up to the metal-vacuum interface. Finally, zone $\mathrm{C}$ constituted the vacuum region. Without any externally applied field, the wavefunctions in Zone $\mathrm{C}$ were plane wave states, while appropriate Airy function solutions were assumed in the presence of an external electric field. The Numerov discretization previously discussed was used for zone B, with the usual continuity of $\psi(z)$ and $d \psi(z) / d z$ at its two boundaries. A representative plot of the electronic wavefunction of $\mathrm{Cu}$ for (100) copper as a function of distance is shown in Fig. 3. This plot was obtained for a normal electron energy $E_{z}$ value of $7.5 \mathrm{eV}$ with an external electric field of $5 \mathrm{GV} / \mathrm{m}$. 

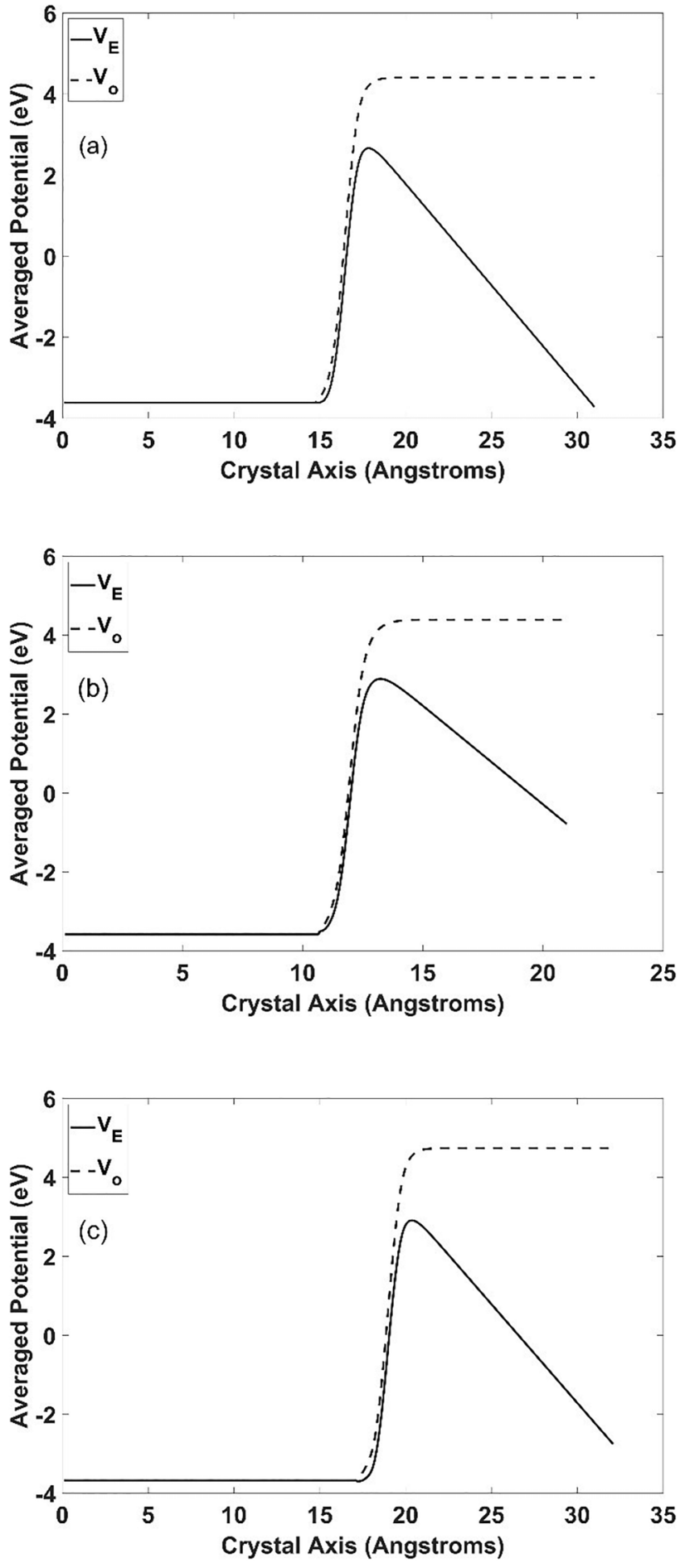

FIG. 2. Position dependent potentials $\overline{\bar{V}}(z)$ at the copper-vacuum boundary for crystalline copper without (dashed lines, $V_{0}$ ) and with (solid lines, $V_{E}$ ) an external electric field of $5 \mathrm{GV} / \mathrm{m}$. Results are shown for the (a) (100) orientation, (b) the (110) direction, and (c) crystalline copper in the (111) orientation.

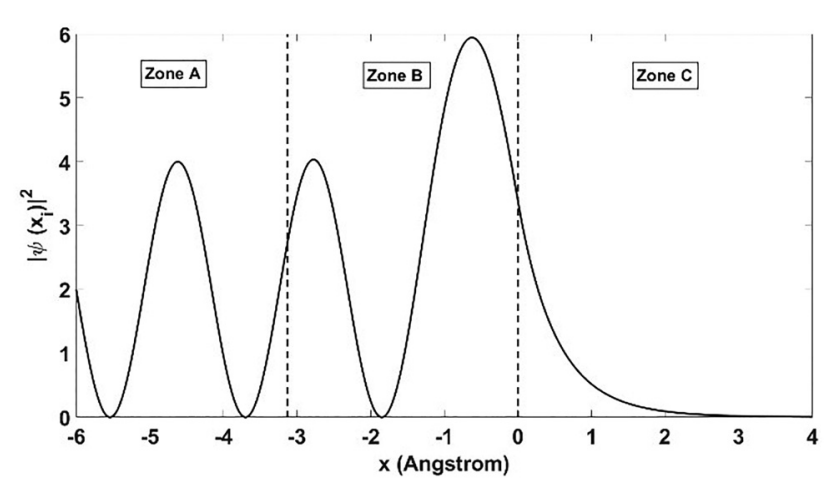

FIG. 3. Plot of $|\psi(z)|^{2}$ as a function of position for the electronic wavefunction of (100) copper with an external field of $5 \mathrm{GV} / \mathrm{m}$ at the normal energy $E_{z}$ of $7.5 \mathrm{eV}$.

Next, predictions of the current density with alterations at the surface, such as the presence of an oxide overlayer, were considered as a natural extension. This is germane and practical, since the electrodes can easily be oxidized. The results for $\overline{\bar{V}}(z)$ obtained from DFT calculations for $\mathrm{Cu}$ (100), $\mathrm{Cu}$ (110), and $\mathrm{Cu}$ (111) surfaces with an oxide are given in Figs. 4(a)-4(c), without (dashed lines, $V_{o}$ ) and with (solid lines, $V_{E}$ ) an external electric field. The adsorption sites for the [100] and [110] directions are similar in that for both directions, the $\mathrm{O}$ atoms prefer to occupy the hollow site as opposed to the top or a bridge site. However, for the [111] direction, the preferred position for the $\mathrm{O}$ atoms is in the octahedral subsurface site between the first and second atomic layers.

The adsorption energy can be used to investigate the adsorption mechanism and energetics between an absorbate and a substrate (bond strength). To better understand the stability and formation of the oxygen ML for different surface orientations, we calculated the adsorption energy. The adsorption energy $\left(E_{a}\right)$ for the surfaces are obtained using

$$
E_{a}=\frac{1}{N}\left(E_{\mathrm{CuO}}-E_{\mathrm{Cu}}-N E_{\mathrm{O}}\right)
$$

where $N$ is the number of $\mathrm{O}$ atoms in the unit cell, $E_{\mathrm{Cu}}$ is the energy of $\mathrm{Cu}, E_{\mathrm{CuO}}$ is the energy of the $\mathrm{CuO}$ system, and $E_{\mathrm{O}}$ is the energy of a free oxygen atom. The calculated adsorption energies include $-4.30 \mathrm{eV},-4.63 \mathrm{eV}$, and $-3.67 \mathrm{eV}$ for the (100), (110), and (111) surfaces, respectively. Interestingly, the work function, which is the difference between the vacuum level (right side of Fig. 4) and the Fermi level located at $0 \mathrm{eV}$, is higher with the oxide than for pure copper. The electronegative nature of oxygen tends to keep the electrons confined to the oxygen, making it more difficult energetically to break free from the surface. This can be directly observed from the adsorption energies of the different surfaces. Increased difficulty in electron extraction and release into the vacuum effectively translates into a higher work function value.

The corresponding transmission coefficient $T\left(E_{z}\right)$ as a function of normal electron energy $E_{z}$ was obtained next. The plot of $T$ $\left(E_{z}\right)$ is given in Fig. 5 for six different cases. For this calculation, the normal external electric field was set at $5 \mathrm{GV} / \mathrm{m}$. Three plots are for 

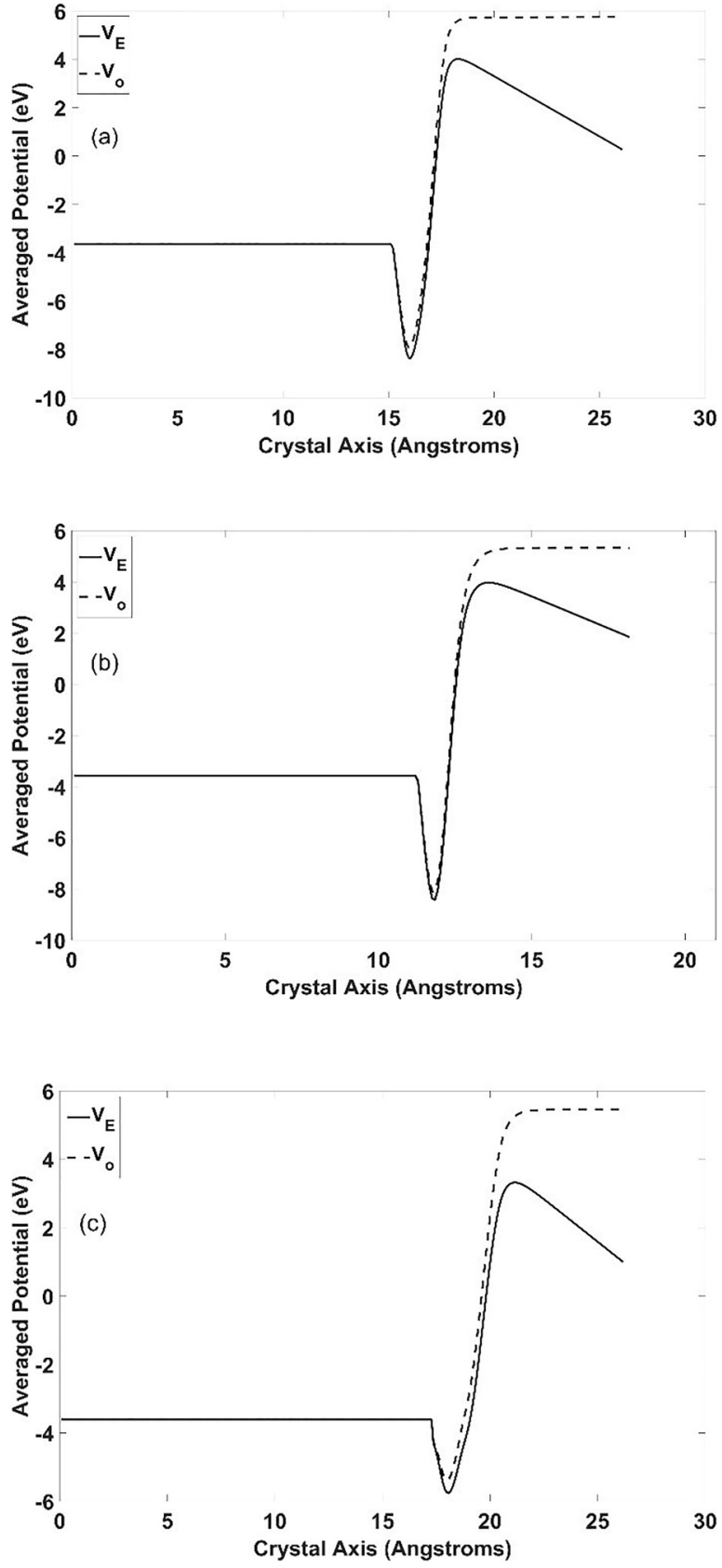

FIG. 4. Position dependent potential for copper with an oxide layer. Results without (dashed lines, $V_{0}$ ) and with (solid lines, $V_{E}$ ) an external electric field of $5 \mathrm{GV} / \mathrm{m}$ are shown for (a) copper along (100) with oxide, (b) copper material along (110), and (c) copper with the (111) orientation.

pure copper for the (100), (110), and (111) orientations, and three other curves are with an oxide layer on the copper surface for the same three orientations (denoted as 100o, 110o, and 111o). As evident from the curves, the tunneling coefficient with the oxide

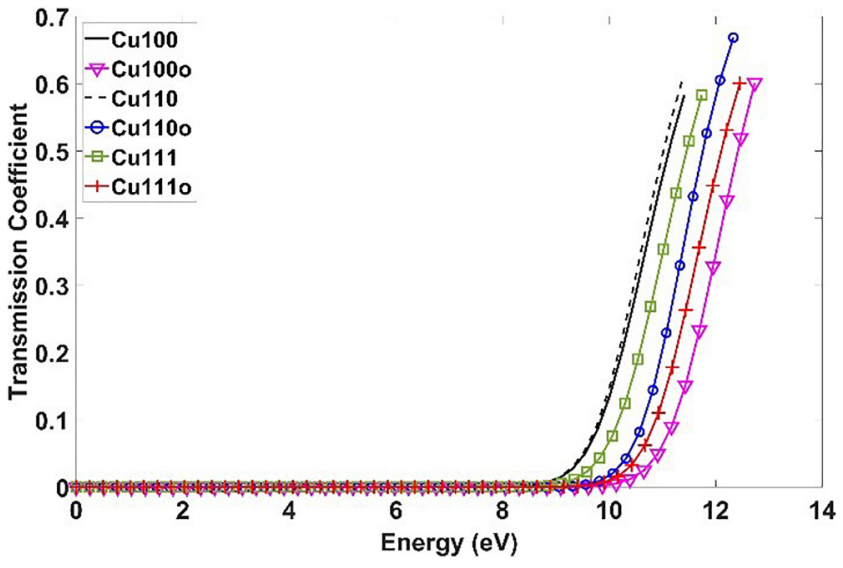

FIG. 5. The transmission coefficient as a function of normal electron energy for six different cases, with and without an oxide layer on the copper. The three crystal orientations of (100), (110), and (111) were chosen. The curves for an oxide layer on the copper surface for the same three orientations are denoted as 1000,1100 , and 1110 . The normal field was set at $5 \mathrm{GV} / \mathrm{m}$.

layer is smaller than that for pure copper for all three orientations. This is qualitatively expected. With an additional oxide layer, the transmission coefficient reduces as electrons coming from deep inside the material onto the copper surface face an additional reflective component at the copper-oxide interface. Any additional mismatch always tends to increase reflection and reduces transmission.

The transmission coefficient obtained was applied for an evaluation of the current density $(J)$ as a function of applied electric field $(F)$. The current density is related to $T\left(E_{z}\right)$ and the Fermi distribution function $f(E)$ as ${ }^{81}$

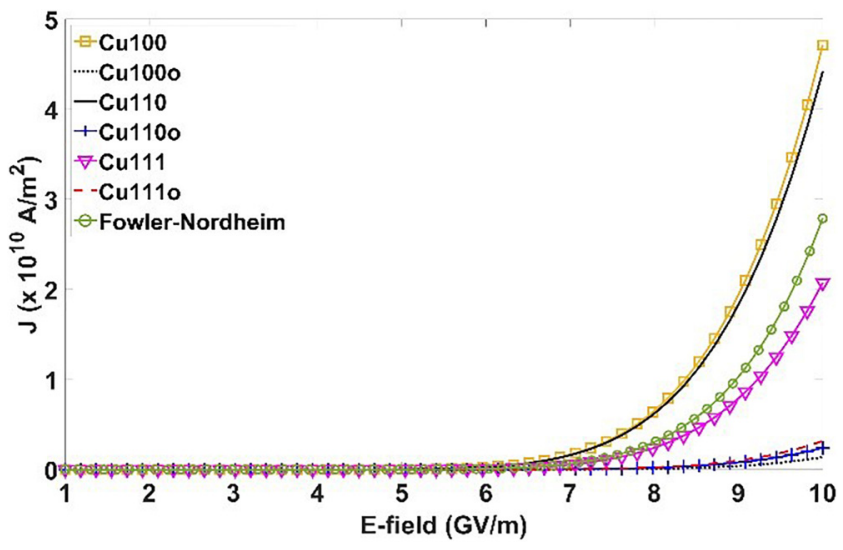

FIG. 6. Electron emission current density $(\Omega$ as a function of external electric field $(F)$ for $\mathrm{Cu}$ and $\mathrm{Cu}-\mathrm{O}$ systems for three different crystal orientations. For comparison, the current density from the usual Fowler-Nordheim model is also shown. 


$$
J=\iiint\left[2 /(2 \pi)^{3}\right] d^{3} k q v_{z} T\left(E_{z}\right) f(E) .
$$

For parabolic energy-wavevector relationships $\left[\hbar^{2} k_{\|}^{2} /(2 m)\right.$ $=E_{\|}$and $\hbar^{2} k_{z}^{2} /(2 m)=E_{z}$ ], the above equation (allowing only the $k_{z}>0$ states and assuming a thermalized Fermi electron distribution function) simplifies to

$$
J_{z}=\left[(q m k T) /\left(4 \pi^{2} \hbar^{3}\right)\right]_{0} \int^{\infty} d E_{z} T\left(E_{z}\right) \ln \left[1+\exp \left(\left(E_{f}-E_{z}\right) /(k T)\right)\right] .
$$

The results are shown in Fig. 6. As evident from the curves, the current density with the oxide layer is smaller than that for
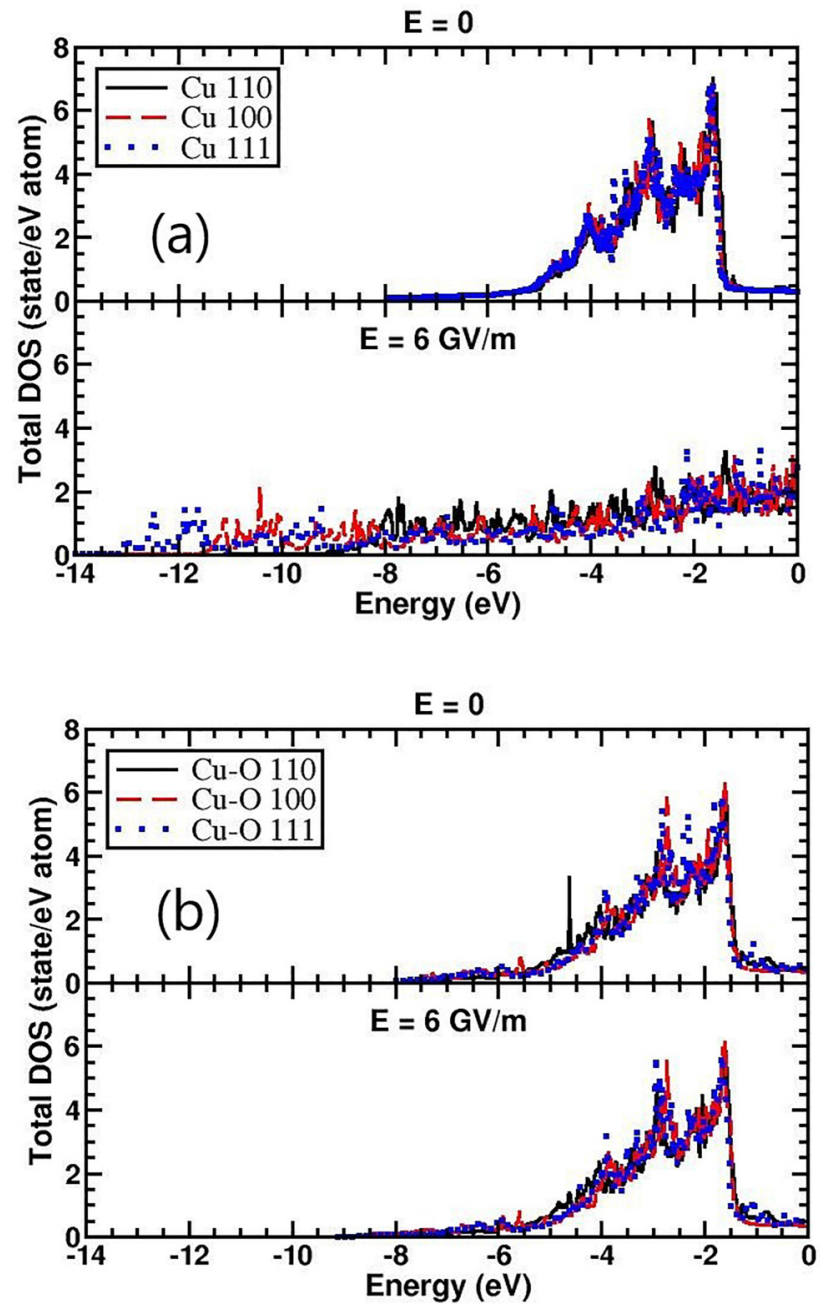

FIG. 7. Calculated total density of states without and with an electric field $E=6 \mathrm{GV} / \mathrm{m}$. The Fermi level is located at $\mathrm{O} \mathrm{eV}$. (a) $\mathrm{Cu}(100), \mathrm{Cu}(110)$, and $\mathrm{Cu}$ (111) surfaces and (b) $\mathrm{Cu}-\mathrm{O}$ (100), $\mathrm{Cu}-\mathrm{O}$ (110), and $\mathrm{Cu}-\mathrm{O}$ (111) surfaces. pure copper for all three orientations. This is qualitatively expected since (i) with an additional oxide layer, the transmission coefficient reduces as already shown as discussed. (ii) Second, the electronegative nature of oxygen, which increases the work function, also leads to reduced current emission. Furthermore, from Fig. 6, it is evident that the electron emission current should be dependent on the surface orientation. Initially, the electric field has little to no effect on the emission current. However, at $\sim 6 \mathrm{GV} / \mathrm{m}$, the surface orientation becomes a factor resulting in $J_{111}<J_{110}<J_{100}$ as the DOS broadening is different and pronounced. For comparison, the current density from the simple Fowler-Nordheim (FN) model is also shown in Fig. 6.

Figures 7(a) and 7(b) show the total density of states (TDOS) for clean $\mathrm{Cu}$ and $\mathrm{Cu}-\mathrm{O}$ surfaces for different orientations without and with an external electric field, respectively. It is clear from Fig. 7 that an applied E field causes a large delocalization and broadening of the TDOS. This broadening is especially prevalent near the Fermi level, indicating that the electrons are able to be excited to the conduction band for all of the orientations. Similar results are reported for studying the surface of a hybrid halide perovskite material. ${ }^{82}$ It is important to mention that the broadening does not only take place near the Fermi level but along the entire spectrum as seen in Fig. 7(b), where the (111) and (110) orientations show the largest and smallest broadening of states, respectively. A shift of the density of states to lower energies under an applied external field causes the (111) orientation to have the smallest current with respect to the other orientations. The trend of TDOS broadening directly affects the electron emission currents.

\begin{tabular}{|c|c|}
\hline Scale: $\Delta \mathrm{n}(\mathrm{r})$ \\
\hline$\square+0.0000$ \\
\hline \\
+0.0000 \\
+0.0000 \\
\hline$\square+0.0000$ \\
\hline$\square+0.0000$ \\
$+\square+0.0001$ \\
$+\square+0.0003$ \\
$+\square+0.0012$ \\
$+\square+0.0047$ \\
$+\square+0.0181$ \\
+0.0694 \\
+0.2664 \\
+1.0224 \\
+3.9245 \\
+15.0631
\end{tabular}
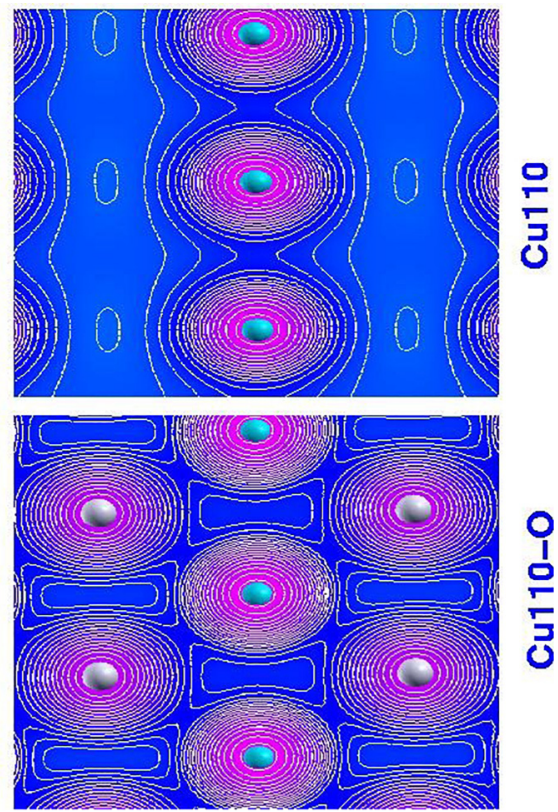

FIG. 8. The charge-density distribution for $\mathrm{Cu}$ (top) and $\mathrm{Cu}-\mathrm{O}$ (bottom) along the [110] direction. Blue spheres are $\mathrm{Cu}$, and gray spheres are $\mathrm{O}$. 
TABLE I. Calculated work functions $(\varphi)$ for $\mathrm{Cu}$ and $\mathrm{Cu}-\mathrm{O}$ surface orientations using GGA. Experimental values are given in parenthesis after Gartland et al. ${ }^{85}$

\begin{tabular}{llc}
\hline \hline Surface & Clean $\mathrm{Cu}(\mathrm{eV})$ & $\mathrm{Cu}-\mathrm{O}(\mathrm{eV})$ \\
\hline$(100)$ & $4.66(4.59)^{85}$ & 5.75 \\
$(110)$ & $4.42(4.48)^{85}$ & 5.50 \\
$(111)$ & $4.75(4.92)^{85}$ & 5.34 \\
\hline \hline
\end{tabular}

It has been shown (Fig. 5) that the addition of an oxygen layer significantly reduced the transmission coefficients for all of the orientations. Studying the TDOS for $\mathrm{Cu}-\mathrm{O}$ surfaces confirms that, contrary to the clean surfaces, a similar external electric field has a smaller effect on the emitted currents. The reason for this lies in the bonding that occurs between the $\mathrm{Cu}$ and $\mathrm{O}$. In order to better understand the role of $\mathrm{O}$ in the broadening of the TDOS, we calculated the charge density of the (110) surface (as an example) with and without the $\mathrm{O}$ layer as shown in Fig. 8. The application of an external electric field causes a polarization of the electrons within the $\mathrm{Cu}$ metal, but this effect is not as dominant in the case of the $\mathrm{Cu}-\mathrm{O}$ material. In Fig. 8 for the charge-density distribution, one can see that the charge along the (110) surface significantly increases when an $\mathrm{O}$ is added to the surface. This is evidence of strong bonding between the $\mathrm{Cu}$ and $\mathrm{O}$ atoms. The $\mathrm{Cu}-\mathrm{O}$ bonding is able to withstand the effects that an external electric field may exert on an oxygenless $\mathrm{Cu}$ metal because of its bond strength. Thus, the electrons in the $\mathrm{Cu}(110)$ surface are allowed to polarize in the presence of an external electric field, whereas for the $\mathrm{Cu}-\mathrm{O}$ (110) surface, the electrons would remain with the $\mathrm{Cu}-\mathrm{O}$ bond resulting in no change in the DOS and little electron emission current. One can extend this to other orientations. Finally, it is important to mention that the (111) $\mathrm{Cu}-\mathrm{O}$ layer has slightly higher density of states closer to the Fermi level, creating a higher emitted current with respect to the other $\mathrm{Cu}-\mathrm{O}$ surface orientations.

For completeness, the calculated and available experimental work functions for clean $\mathrm{Cu}$ and $\mathrm{Cu}-\mathrm{O}$ surfaces orientations are given in Table I. For clean surfaces, the calculated work functions are within $3.7 \%$ of experimental measurements. The addition of oxygen is predicted to increase the work function significantly. This large change of work function can be explained based on the nature of the strong bond between $\mathrm{Cu}$ and $\mathrm{O}$ atoms as was discussed before.

\section{SUMMARIZING CONCLUSIONS}

Field emission is an important process with applications toward the development of bright electron sources for high-resolution electron microscopes, for charged particle generation from microwave devices, lithography, electronic displays, and some medical therapeutics. Quantitative predictions need to include details of the internal potentials that shape the electronic wavefunctions (and hence the tunneling probability); details of work function barrier $\left(\Phi_{B}\right)$ at the boundary that is dependent on material quality and lattice structure including possible defects; and values of the local density of states (DOS) at the surface that influence the supply of electrons. Here, these various factors were collectively included based on density functional theory, to obtain quantitative predictions of fielddependent electron tunneling current densities.

Results were obtained in copper oriented along three different directions. The density of states (DOS) was predicted to be broadened by an externally applied electric field. The (111) and (110) orientations for copper showed the largest and smallest broadening of states, respectively. The largest current density was obtained for (100) copper, while the lowest was predicted for the (111) orientation. In the presence of an oxide layer at the surface, the work function increased significantly, leading to much smaller emission currents. Such calculations will aid in the design assessment of cathodes for high power microwave devices. The technique present here is general and can be applied to other materials such as carbon nanotubes or carbon fibers that have shown promise as superior candidates for cathode emitters. For realistic emitters having conical, ellipsoidal, spheroidal, or other shapes, a three-dimensional analysis would be necessary with adequate inclusion of lateral changes in the work function and screened electric fields $s^{51,83,84}$ at the emitting tip. Although the role of defects was not studied, this method could be used to analyze systems containing vacancies and/or substitutional defects. However, when defects are included in the present formulation, a larger unit cell would be needed to avoid any inadvertent or artificial enhancements in the defect density.

\section{ACKNOWLEDGMENTS}

This work was supported, in part, by Grants from the Office of Naval Research (No. N00014-18-1-2382) and the Air Force Office of Scientific Research (No. FA9550-19-1-0056).

\section{DATA AVAILABILITY}

The data that support the findings of this study are available from the corresponding author upon reasonable request.

\section{REFERENCES}

${ }^{1}$ R. H. Fowler and L. Nordheim, Proc. R. Soc. London A 119, 173 (1928).

${ }^{2}$ R. H. Fowler, Proc. R. Soc. London A 117, 549 (1928)

${ }^{3}$ L. W. Nordheim, "Die Theorie der Elektronenemission der Metalle," Z. Phys. 30, 177 (1929).

${ }^{4}$ W. W. Doland and W. Dyke, Phys. Rev. 95, 327 (1954).

${ }^{5}$ W. P. Dyke and J. K. Trolan, Phys. Rev. 89, 799 (1953).

${ }^{6}$ R. G. Forbes, Ultramicroscopy 79, 11 (1999).

${ }^{7}$ R. G. Forbes, Surf. Interface Anal. 36, 395 (2004).

${ }^{8}$ K. L. Jensen, J. Appl. Phys. 126, 065302 (2019).

${ }^{9} \mathrm{~K}$. L. Jensen, Introduction to the Physics of Electron Emission (John Wiley \& Sons, Inc, Hoboken, NJ, 2017).

${ }^{10} \mathrm{X}$. Calderon-Colon, H. Geng, B. Gao, L. An, G. Cao, and O. Zhou, Nanotechnology 20, 325707 (2009).

${ }^{11}$ C. A. Spindt, C. E. Holland, P. R. Schwoebel, and I. Brodie, J. Vac. Sci. Technol. B 16, 758 (1998).

${ }^{12}$ G. Cao, Y. Z. Lee, R. Peng, Z. Liu, R. Rajaram, X. Calderon-Colon, L. An, P. Wang, T. Phan, S. Sultana, D. S. Lalush, J. P. Lu, and O. Zhou, Phys. Med. Biol. 54, 2323 (2009).

${ }^{13}$ R. J. Barker and E. Schamiloglu, High-power Microwave Sources and Technologies (Wiley-IEEE Press, New York, 2001). 
${ }^{14}$ J. H. Booske, R. J. Dobbs, C. D. Joye, C. L. Kory, G. R. Neil, G. S. Park, J. Park, and R. J. Temkin, IEEE Trans. Terahertz Sci. Technol. 1, 54 (2011).

${ }^{15}$ Y. Chen, Microelectron. Eng. 135, 57 (2015).

${ }^{16}$ J. E. Polk, M. J. Sekerak, J. K. Ziemer, J. Schein, N. Qi, and A. Anders, IEEE Trans. Plasma Sci. 36, 2167 (2008)

${ }^{17}$ L. Nilsson, O. Groning, C. Emmenegger, O. Kuettel, E. Schaller, L. Schlapback, H. Kind, J. M. Bonard, and K. Kern, Appl. Phys. Lett. 76, 2071 (2000).

${ }^{18}$ J. S. Suh, K. S. Jeong, J. S. Lee, and I. Han, Appl. Phys. Lett. 80, 2392 (2002).

${ }^{19}$ Y. Li, Y. Sun, and J. T. W. Yeow, Nanotechnology 26, 242001 (2015)

${ }^{20}$ Z. Zhang, G. Meng, Q. Wu, Z. Hu, J. Chen, Q. Xu, and F. Zhou, Sci. Rep. 4, 4676 (2015).

${ }^{21}$ J. R. Oppenheimer, Phys. Rev. 31, 66 (1928).

${ }^{22}$ R. W. Gurney and E. U. Condon, Nature 122, 439 (1928).

${ }^{23} \mathrm{G}$. Gamow, Nature 122, 805 (1928).

${ }^{24}$ C. Zener, Proc. R. Soc. London A 145, 523 (1934).

${ }^{25}$ A. J. Heeger, Angew. Chem. Int. Ed. Engl. 40, 2591 (2001).

${ }^{26}$ G. R. Hutchison, M. A. Ratner, and T. J. Marks, J. Phys. Chem. B 109, 3126 (2005).

${ }^{27}$ E. A. Weiss, M. R. Wasielewski, and M. A. Ratner, "Molecular wires from design to properties," in Topics in Current Chemistry, edited by L. DeCola (Springer, Berlin, 2005), Vol. 257, pp. 103-133.

${ }^{28}$ M. Ratner, Nat. Nanotechnol. 8, 378 (2013).

${ }^{29} \mathrm{~N}$. Renaud, M. Hliwa, and C. Joachim, "Unimolecular and supramolecular electronics II chemistry and physics meet at metal-molecule interfaces," in Topics in Current Chemistry, edited by R. M. Mezger (Springer, Berlin, 2012), Vol. 313, pp. 217-268.

${ }^{30}$ J. R. Winkler and H. B. Gray, J. Am. Chem. Soc. 136, 2930 (2014).

${ }^{31}$ O. W. Richardson and A. F. A. Young, Proc. R. Soc. London A 107, 377 (1925).

${ }^{32}$ E. L. Murphy and R. H. Good, Phys. Rev. 102, 1464 (1956).

${ }^{33}$ C. Herring and M. H. Nichols, Rev. Mod. Phys. 21, 185 (1949).

${ }^{34}$ Y. Feng and J. P. Verboncoeur, Phys. Plasmas 12, 103301 (2005).

${ }^{35}$ R. G. Forbes, Appl. Phys. Lett. 89, 113122 (2006).

${ }^{36}$ J. H. B. Deane and R. G. Forbes, J. Phys. A Math. Theor. 41, 395301 (2008).

${ }^{37}$ K. L. Jensen, J. Vac. Sci. Technol. B 21, 1528 (2003).

${ }^{38} \mathrm{~K}$. Jensen and M. Cahay, Appl. Phys. Lett. 88, 154105 (2006).

${ }^{39}$ K. L. Jensen, J. Appl. Phys. 102, 024911 (2007).

${ }^{40}$ L. I. Schiff, Quantum Mechanics, 3rd edition (McGraw-Hill, New York, 1970), Chap. 34

${ }^{41}$ K. L. Jensen, J. Appl. Phys. 85, 2667 (1999).

${ }^{42}$ N. D. Lang, A. Yacoby, and Y. Imry, Phys. Rev. Lett. 63, 1499 (1989).

${ }^{43}$ P. Hohenberg and W. Kohn, Phys. Rev. 136, B864 (1964); W. Kohn and L. J. Sham, ibid. 140, A1133 (1965).

${ }^{44}$ Y. Gohda, Y. Nakamura, K. Watanabe, and S. Watanabe, Phys. Rev. Lett. 85, 1750 (2000)

${ }^{45}$ W. Tang, D. Shiffler, and K. L. Cartwright, J. Appl. Phys. 110, 034905 (2011).

${ }^{46}$ R. Miller, Y. Y. Lau, and J. H. Booske, J. Appl. Phys. 106, 104903 (2009).

${ }^{47}$ H. Qiu, R. P. Joshi, A. Neuber, and J. Dickens, Semicond. Sci. Technol. 30, 105038 (2015).

${ }^{48}$ D. Biswas, Phys. Plasmas 25, 043113 (2018).

${ }^{49}$ R. G. Forbes and J. H. B. Deane, Proc. R. Soc. A 463, 2907 (2007).

${ }^{50}$ J. Lin, P. Y. Wong, P. Yang, Y. Y. Lau, W. Tang, and P. Zhang, J. Appl. Phys. 121, 244301 (2017)
${ }^{51}$ K. L. Jensen, D. A. Shiffler, J. R. Harris, and J. J. Petillo, AIP Adv. 6, 065005 (2016); K. L. Jensen, M. McDonald, O. Chubenko, J. R. Harris, D. A. Shiffler, N. A. Moody, J. J. Petillo, and A. J. Jensen, J. Appl. Phys. 125, 234303 (2019).

${ }^{52}$ C. A. Spindt, I. Brodie, L. Humphrey, and E. R. Westerberg, J. Appl. Phys. 47, 5248 (1976).

${ }^{53}$ R. V. Latham, High-Voltage Vacuum Insulation: Basic Concepts and Technological Practice (Academic Press, London, 1995).

${ }^{54}$ H. Andrews, K. Nichols, D. Kim, E. I. Simakov, S. Antipov, G. Chen, M. Conde, D. Doran, G. Ha, W. Liu, J. Power, J. Shao, and E. Wisniewski, IEEE Trans. Plasma Sci. 48, 2671 (2020).

${ }^{55}$ T. Cao, L. Luo, Y. Huang, B. Ye, J. She, S. Deng, J. Chen, and N. Xu, Sci. Rep. 6, 33983 (2016).

${ }^{56}$ V. I. Kleshch, V. A. Eremina, P. Serbun, A. S. Orekhov, D. Lützenkirchen-Hecht, E. D. Obraztsova, and A. N. Obraztsov, Phys. Status Solidi B 255, 1700268 (2018).

${ }^{57}$ W. Lei, C. Li, M. T. Cole, K. Qu, S. Ding, Y. Zhang, J. H. Warner, X. Zhang, B. Wang, and W. I. Milne, Carbon 56, 255 (2013).

${ }^{58}$ T. Utsumi, IEEE Trans. Electron Devices 38, 2276 (1991).

${ }^{59}$ Y. B. Zhu, P. Zhang, A. Valfells, L. K. Ang, and Y. Y. Lau, Phys. Rev. Lett. 110, 265007 (2013).

${ }^{60}$ Y. B. Zhu and L. K. Ang, Phys. Plasmas 22, 052106 (2015).

${ }^{61}$ W. D. Kesling and C. E. Hunt, IEEE Trans. Electron Devices 42, 340 (1995).

${ }^{62}$ J. J. Petillo, E. M. Nelson, J. F. Deford, N. J. Dionne, and B. Levush, IEEE Trans. Electron Devices 52, 742 (2005).

${ }^{63}$ K. L. Jensen, D. A. Shiffler, M. Peckerar, J. R. Harris, and J. J. Petillo, J. Appl. Phys. 122, 064501 (2017).

${ }^{64} \mathrm{M}$. Finnis, Interatomic Forces in Condensed Matter (Oxford University Press, New York, 2003).

${ }^{65}$ J. M. Blatt, J. Comput. Phys. 1, 382 (1967).

${ }^{66}$ P. C. Chow, Am. J. Phys. 40, 730 (1972).

${ }^{67}$ R. H. Landau, M. J. Paez, and C. C. Bordeianu, A Survey of Computational Physics (Princeton University Press, Princeton, NJ, 2008).

${ }^{68}$ See https://www.vasp.at/ for more information about the VASP simulator.

${ }^{69}$ G. Kresse and J. Hafner, Phys. Rev. B 47, 558 (1993).

${ }^{70} \mathrm{G}$. Kresse and J. Furthmuller, Phys. Rev. B 54, 11169 (1996).

${ }^{71}$ P. E. Blöchl, Phys. Rev. B 50, 17953 (1994).

${ }^{72}$ G. Kresse and D. Joubert, Phys. Rev. B 59, 1758 (1999).

${ }^{73}$ J. P. Perdew, K. Burke, and M. Ernzerhof, Phys. Rev. Lett. 77, 3865 (1996).

${ }^{74}$ J. Neugebauer and M. Scheffler, Phys. Rev. B 46, 16067 (1992).

${ }^{75}$ G. Makov and M. C. Payne, Phys. Rev. B 51, 4014 (1995).

${ }^{76}$ P. E. Blöchl, O. Jepsen, and O. K. Andersen, Phys. Rev. B 49, 16223 (1994).

${ }^{77}$ F. D. Murnaghan, Proc. Natl. Acad. Sci. U.S.A. 30, 244 (1944).

${ }^{78}$ G. Makov and M. C. Payne, Phys. Rev. B 51, 4014 (1995).

${ }^{79}$ L. Bengtsson, Phys. Rev. B 59, 12301 (1999).

${ }^{80} \mathrm{~V}$. Wang, N. Xu, J. Liu, G. Tang, and W. Geng, "VASPKIT: A pre- and postprocessing program for VASP code,” arXiv:1908.08269 (2019).

${ }^{81}$ C. B. Duke, Tunneling in Solids (Academic Press, New York, 1969).

${ }^{82}$ D. Ji, M. Na, S. Wang, H. Zhang, K. Zhu, C. Zhang, and X. Li, Sci. Rep. 8, $12492(2018)$

${ }^{83}$ D. A. Shiffler, W. Tang, K. L. Jensen, K. Golby, M. LaCour, J. J. Petillo, and J. R. Harris, J. Appl. Phys. 118, 083302 (2015).

${ }^{84}$ W. Tang, D. Shiffler, and K. L. Cartwright, J. Appl. Phys. 110, 034905 (2011).

${ }^{85}$ P. O. Gartland, S. Berge, and B. J. Slagsvold, Phys. Rev. Lett. 28, 738 (1972). 